\title{
Study on Plasmid Curing Towards Environmental Application
}

\author{
Badrunnisa. $\mathrm{S}^{1}$, Sanjay Deshpande ${ }^{2}$, Aiswarya. $\mathrm{R}^{3}$, H J Sumana ${ }^{4}$, Giridhar ${ }^{5}$ \\ ${ }^{1}$ BITM Bellary, Karnataka, India, ${ }^{2}$ BITM Bellary, Karnataka, India, ${ }^{3}$ BITM Bellary, Karnataka, India, ${ }^{4}$ BITM Bellary, \\ Karnataka, India, ${ }^{5}$ BITM Bellary, Karnataka, India,
}

\begin{abstract}
Oil spill have a huge impact towards the destruction of environment and also in causing ecological imbalance, hence an efficient technique has to be found for the removal of oil spills efficiently. Some microorganisms belonging to the pseudomonas family like Pseudomonas aeruginosa, Pseudomonas putida have a capacity to degrade wide variety of complex carbon compounds, but the pathogenic nature make them unfit for the direct usage for the removal of oil spills. This study attempts to transfer the $x y l$ gene incorporated in plasmid of Pseudomonas aeruginosa to a novel organism like Bacillus thuringiensis, Bacillus cereus which are devoid of the degradative character. In conclusion this novel organism can be used towards the oil spill treatments, thereby protecting the environment and the health of workers.
\end{abstract}

\section{Keywords:- Bacillus thuringiensis, Plasmid and Pseudomonas aeruginosa}

\section{INTRODUCTION}

Plasmid is an extra chromosomal genetic material that occurs in most of bacterial strains. They are usually found in bacteria. Plasmid sizes vary from 1 to over $1,000 \mathrm{kbp}$ [1] Plasmid host-to-host transfer requires direct, mechanical transfer by conjugation or changes in host gene expression allowing the intentional uptake of the genetic material by transformation. One way of classify plasmids is by their ability to transfer to other bacteria. Another way to classify plasmids is by function. There are five main classes: Fertility F-plasmids, Resistance (R) plasmids, Col plasmids, Degradative plasmids, Virulence plasmid [1].

Pseudomonas aeruginosa is increasingly recognized as an emerging opportunistic pathogen of clinical relevance. Several different epidemiological studies indicate antibiotic resistance is increasing in clinical isolates $[2,3,4,5]$.

Biofilms formation: Pseudomonas has the ability to metabolize a variety of diverse nutrients. Combined with the ability to form biofilms [5, 6], they are thus able to survive in a variety of unexpected places. For example, they have been found in areas where pharmaceuticals are prepared. A simple carbon source, such as soap residue or cap liner-adhesives is a suitable place for them to thrive. Other unlikely places where they have been found include antiseptics, such as quaternary ammonium compounds, and bottled mineral water.

Pseudomonas putida is a gram-negative rodshaped saprotrophic soil bacterium. It is the first patented organism in the world. It demonstrates a very diverse metabolism, including the ability to degrade organic solvents such as toluene. This ability has been put to use in bioremediation, or the use of microorganisms to biodegrade oil. Use of $P$. putida is preferable to some other Pseudomonas species capable of such degradation as it is a safe species of bacteria, unlike $P$. aeruginosa for example, which is an opportunistic human pathogen.

Bacillus thuringiensis is a Gram-positive, soildwelling bacterium, commonly used as a biological pesticide; alternatively, the Cry toxin may be extracted and used as a pesticide. Bacillus thuringiensis also occurs naturally in the gut of caterpillars of various types of moths and butterflies, as well as on the dark surfaces of plants [3, 6]. During sporulation, many Bacillus thuringiensis strains produce crystal proteins (proteinaceous inclusions), called $\delta$-endotoxins, that have insecticidal action. This has led to their use as insecticides, and more recently to genetically modified crops using Bacillus thuringiensis genes.

There are, however, many crystal-producing Bacillus thuringiensis strains that do not have insecticidal properties $[7,8]$.

Present study attempts to isolate plasmid from opportunistic pathogen, identify and characterize using bioinformatics tools such as NCBI, EBI, Entrez, Pair-wise alignment, COPICAT and transfer the biodegrading ability of Pseudomonas aeruginosa to Bacillus cereus towards biofilm treatment $[9,10]$.

\section{METHODOLOGIES}

\subsection{Collection of organisms}

Test organisms Pseudomonas aeruginosa, Bacillus thuringiensis and Bacillus cereus were collected from Microbiology Lab, BITM, Bellary.

\subsection{Culture maintenance}

Collected organisms were sub-cultured every 48 hours on nutrient broth and agar plate and were used for further studies.

\subsection{Plasmid isolation}

The pure culture of Pseudomonas aeruginosa was taken into sterile fresh vial, to the full volume of the vial, centrifuged at $6000-8000 \mathrm{rpm}$ for about $10-20$ minutes. The pellets were dissolved in $100 \mu \mathrm{l}$ solution I and incubated for 10 minutes in ice cold conditions followed by the addition of $200 \mu \mathrm{l}$ of solution II, incubated at room temperature and then placed on ice for 15 minutes with the addition of $150 \mu \mathrm{l}$ of solution III. Centrifuged and $450 \mu \mathrm{l}$ of solution IV was added to supernatant and dried at $37^{\circ} \mathrm{C}$ for $10-15$ minutes, 
finally stored at $4^{\circ} \mathrm{C}$ with $1 \mathrm{X}$ TE buffer for further confirmation $[11,12]$.

\subsection{Sequence retrieval}

Sequence retrieval is carried out using NCBI-Entrez database. A query for Pseudomonas aeruginosa is given and once the search is completed, many results are displayed in that select Genome. Once the genome is selected many genomic sequences including the plasmid sequence are displayed in that select plasmid genome and select the FASTA format to visualize the Plasmid sequence. The required gene sequence can be obtained using various databases in that the primary and best databases is NCBI database and give the query of the xyl gene which is the carbon catabolic gene and retrieve the FASTA sequence for the further studies [13].

2.5 Comparison between plasmid and gene sequence Comparative studies of the sequences of Pseudomonas aeruginosa plasmid and Pseudomonas putida gene was carried out. The sequences were retrieved in the FASTA format from NCBI website using Entrez Go to EMBOSS ALIGN Home page. In the submission form, copy and paste the sequences in the space provided. Run the job with the EMBOSS with the local alignment program (water) using the two sequences. Once the job is completed the result is given in the form of an alignment file [14].

\subsection{Chemical and binding relationship with proteins}

A Chemical and its binding relationship with proteins is carried out using a online tool called COPICAT (Comprehensive Predictor of Interactions between Chemical compounds And Target proteins).In this tool the chemical compound is uploaded in varies forms like PubChem Id, sdf file format .The protein input is given in the form of FASTA file or else the Uniprot Id is given and multiple entries are also accepted. After the chemical and protein inputs are submitted the job is run and the output will be displayed after a certain period of time. The result is obtained in the form of CSV file which contain the result in the form of probability measure i.e. probability of chemical binding to the protein given. Based on the values of probability the possibility of binding can be concluded [15, 16].

\subsection{Transformation to Bacillus thuringiensis}

Transformation of bacillus thuringiensis with Sequences of the required gene were retrieved by using Pseudomonas aeruginosa is carried out using heat shock meflofire 2).

The transformation process is mainly carried out in two steps first

step to process the competent cells that are ready to uptakg.5heomparison between plasmid and gene sequence plasmid and this is done by using Calcium chloride treatmenCanparison of the plasmid sequence of Pseudomonas second step in transformation process that is transfer of plasmiquatda and retrieved gene sequence was done by using EBI the competent cells by heat shock method. For the competentPallwise sequence alignment. Results shows upto $40 \%$ preparation first chill the culture flask for 20 minutes and transfestithiłarity between Pseudomonas aeruginosa and chilled culture to vials aseptically after the cultures are transferseddomonas putida plasmid sequence (Figure 3 ). into vials centrifuge the vials at 6000rpm for 8 minutes, collect the pellets in a fresh vial and add $1500 \mu \mathrm{l}$ of Calcium chloride to the.6emical and binding relationship with proteins pellets and place on ice for 30 minutes and the centrifuge at 6000rmpound binding and its Probability study was carried out for 8-10 min. Discard the supernatant, resuspend the cell pelletsinin COPICAT tool. The results obtained were -1 which $600 \mu \mathrm{l}$ of ice cold Calcium chloride and competent cells are reaiflydieates that the type of binding is reversible and the $\mu \mathrm{l}$ of plasmid DNA is added to $100 \mu \mathrm{l}$ of competent cells. Mijrdbrability of binding is 0.12 (Figure 4).

gently tapping and incubate for 20 minutes. After the incubation give 


\subsection{Transformation}

Heat shock method as mentioned earlier was used for the transformation of Bacillus thuringiensis and the presence of the transformed gene was confirmed both qualitatively and quantitatively (Figure 5, 6, 7\&8).

\subsection{Confirmation Tests}

A noticeable reduction in the layer of oil present in the culture flask inoculated with transformed Bacillus thuringiensis was observed whereas the flask inoculated with transformed Bacillus cereus did not show the reduction in the oil layer. This infers that the oil degrading plasmid is been expressed markably in Bacillus thuringiensis compared to Bacillus cereus (Figure 9, 10\&11).

Using drop-weight method the surface tension values were calculated where there was a noticeable increase in the surface tension values of broth just after inoculation as well as after a certain period of incubation.

Optical density was calculated for both the samples where $27.68 \%$ degradation of oil was found by 968 cells in 69 hours (Table 1 and Figure 13).

\section{FIGURES AND TABLES}

Pseudomonas aeruginosa plasmid pBS228, complete sequence NCBI Reference Sequence: NC_008357.1

\section{GenBank Graphics}

>gi|114881096|ref|NC_008357.1| Pseudomonas aeruginosa plasmid pBS228, complete sequence

TTCACCCCCGAACACGAGCACGGCACCCGCGACCACTATGCCAAGAATGCCCAAGGTAAAAATTGCCGGC CCCGCCATGAAGTCCGTGAATGCCCCGACGGCCGAAGTGAAGGGCAGGCCGCCACCCAGGCCGCCGCCC CACTGCCCGGCACCTGGTCGCTGAATGTCGATGCCAGCACCTGCGGCACGTCAATGCTTCCGGGCGTCGC GCTCGGGCTGATCGCCCATCCCGTTACTGCCCCGATCCCGGCAATGGCAAGGACTGCCAGCGCCGCGAT AGGAAGCGGGTGCCCCGCTTCTTCATCTTCGCGCCTCGGGCCTCGAGGCCGCCTACCTGGGCGAAAACAT CGGTGTTTGTGGCATTCATACGGACTCCTGTTGGGCCAGCTCGCGCACGGGCTGGCGGGTCAGCTTGGCI TGAAGATCGCCACGCATTGCGGCGATCTGCTTCTCGGCATCCTTGCGCTTCTGCACGCCTTCCTGCTGG TGCGAATAACGTCCTCGACGGTCTTGATGAGCGTCGTCTGAACCTGCTTGAGCGTGTCCACGTCGATCAC CAGGCGTTGGTTCTCCTTCGCCGTCTCGACGGACGTGCGATGCAGCAGGGCCGCATTGCGCTTCATCAGG TCGTTGGTGGTGTCGTCGATGGCCGTGGCCAGTTCGACGGCGTTCTTCTGCTCGTTGAGGCTCAAGGCC GCATGAATTGCCGCTTCCACGCCGGCACGGTGATTTCGCGGATGGTGTGgaATTTATCGACCAGCATCT GTTGTTGGCCTGGATCATGCGGATGGTCGGCAGGCTCTGCATGGCCGAATGTTGCAAGGCGATCAGGTCG CCGATGCGCTTGTCCAGGTTGGCAACCATCGCATCGAGGTCGGCCAGCTCCTGCACGCGGCCCGGGTCGI TCCCGACATTGCCGCGCAGACCCTCGGCCTGCTCGCGCAGCTCGGCAAGGCGGACCTTGCCGGCCGCGAT GTGGACGCCAAGAAGGCGGTGTTCCTCGCGCACGGCTGCGAACATTTCGTCGAGCGAGGCATTGCGCTGC GCGATGCCTTGCTGGGTGGTCTGCACTTCGCTGACCAGGTGTTCGATCTGCTCGCGGGTCGIGTCGAAGC GCGCCATGAAGCCCGTCGAACGGACGCGGAAGCGGTCGATCAGCGGGCCAATCAGGGGCAGGCGGGAACG GTTGTCGGACAAAGGGCCGACGTTCAGGGAACGGGCCTTGGCGACAACCTGGGTCAGTTTCTCGCCTGCT TCGTCCAGGTCGCTGTTGCGCACCTGGTCCAGCAGGCTATCGGCGTAGCGGGACGTGTGCTCGGCCACGI CGCGGCCGAACTCGGCAACGGTCTGCGGACTGCCGACCTCGATCCGCTGCGCGACCGCATGGACTTCCGG

Fig 1: Plasmid sequence of Pseudomonas aeruginosa.

\section{Pseudomonas putida plasmid pWW0, complete sequence}

NCBI Reference Sequence: NC_003350.1

GenBank Granhics

>gi|18150858:c43137-41437 Pseudomonas putida plasmid pWWo, complete sequence ATGICGCTTACATACAAACCCAAGATGCAGCATGAGGATATGCAAGACCTTAGCAGCCAGATCCGTTTCG TTGCCGCCGAAGGCAAGATCTGGTTGGGAGAGCAGCGCATGCTCGTAATGCAGCTATCTACGCTGGCCA CTTCCGICGCGAAATTATCAGCTTGATCGGCGICGAGCGGGCCAAGGGITTCTICCTGCGGITGGGCTAT CAGTCCGGCCTGATGGATGCCGAGCIGGCACGCAAGCTGCGGCCGGCCATGCGCGAGGAGGAGGIGTTCC IGGCTGGGCCTCA.ATTGTATGCGCTCAAGGGGATGGTCAAAGTACGCTIGCTGACA.ATGGATATCGCCAT CCGGGACGGACGITTCAACGIGGAGGCCGAGIGGATTGATTCCTTTGAAGTGGATATCTGCCGAACTGAG CIGGGCCTGATGAAIGAGCCCGICTGCTGGACGGIGCTAGGCTATGCTAGCGGCTATGGTTCGGCATTCA IGGGCCGCAGAATCATTTTCCAGGAAACTAGCTGICGCGGGTGCGGTGACGATAAATGCCTTATCGTCGG CAAGACCGCAGA. GA GIGGGGCGATGICAGCAGTTTCGAAGCCTACTTCAAAAGCGACCCGATCGTAGAC GAGCGCTACGAGCTGCAGACCCAGGTTGCCAACCTGCGCAACCGCCTGAAGCAGTACGATGGGCAGTATACGGCATTGGCCATTCGCCAGCCTACAAGCGCATCTGIGAGACCATCGACAAGGCTGCACGCGGCAGGGI ITCGGTCCTGCTACTGGGTGAGACTGGGGTGGGCAAGGAGGTAATCGCGCGCAGCGTGCATTTGCGCAG GAGCGCGCAGAGCAACCCITCGTCGCGGIGAACTGTGCGGCAATTCCGCCGGATCTGATCGAGTCGGAAC TGTTTGGTGTCGATAAGGGCGCCTATACGGGCGCGGTCAATGCACGCGCTGGACGTITTGAACGGGCCAR CGGCGGCACCATCTITCTTGATGAGGTGATCGAATTGACGCCGAGGGCCCAGGCCACCCTGCTACGGGT TTGCAGGAAGGAGAGCTAGAGCGGGTCGGCGGCGACCGCACGCGAAAGGTCGACGTGAGGTTAATCACCG CAACAAACGAGAACCTGGAAGAGGCGGTCAAGATGGGGCGCTTTCGCGCAGACCTGITCITTCGGCTGA IGTTTTTCCCGIGCATATCCCGCCGITGCGCGAGCGCGTGGAAGATATCCCGCTGCTGGICGAGCATTTI CTTAGA.AGGCACCATARGGAATACGGTAAGA.AGACTCTTGGCCTGICTGATCGAGCGATGGAGGCCIGCC TCCACTACCAATGGCCAGGCAATATCCGCGAGCTGGAGAACGCCCTIGAGCGCGGGGIGATTCTTACCGA GAGCAACGAAAGCATCAATGTCGAGTCGCTGTTCCCGGGGTTGGCGACGGCTACCGAAGGCGACAGGCT ICGAGCGAGGGCCGGITGGAGGAGGAGTCCGGTGACAGITGGTTTAGGCAAATTATCGACCAGGGCGTCA GCCTCGAAGATCTCGA.AGCGGGTTTAATGCGCACGGCCATGGACCGTTGIGGGCAGAATATCTCACAGGC GGCGCGGITGCTGGGATIGACCCGCCCGGCAAIGGCCTATCGACTTAAGAAGCTTGACCCCAGCTTATCI GIGAARGCAATGGGCCGATAG

Fig 2: Complete sequence of Pseudomonas putida plasmid pWW0.

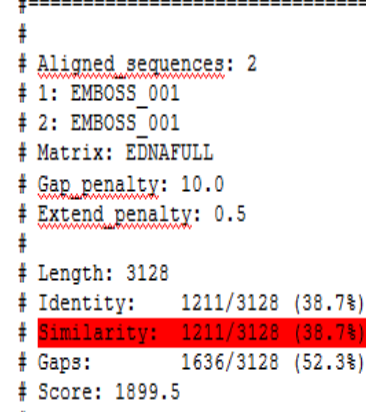

Fig 3: Pairwise alignment studies between Pseudomonas aeruginosa and Pseudomonas putida displaying results of similarity of about $40 \%$.

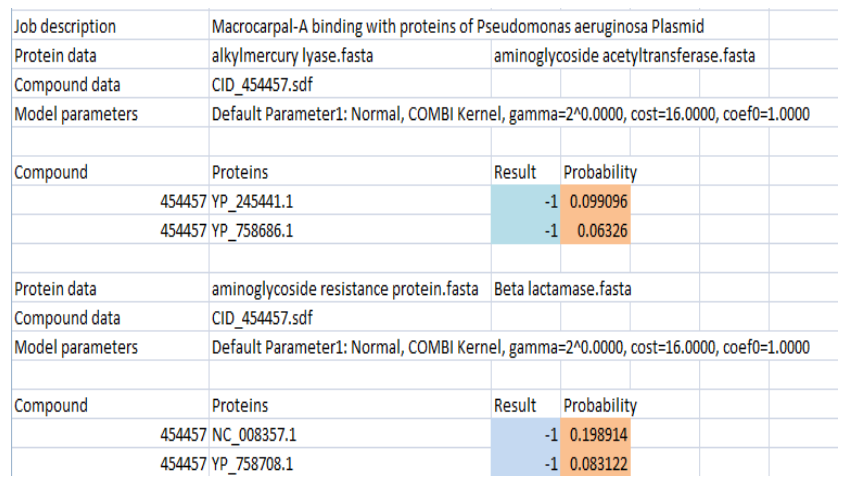

Fig 4: Compound binding and its Probability study results using COPICAT tool. 


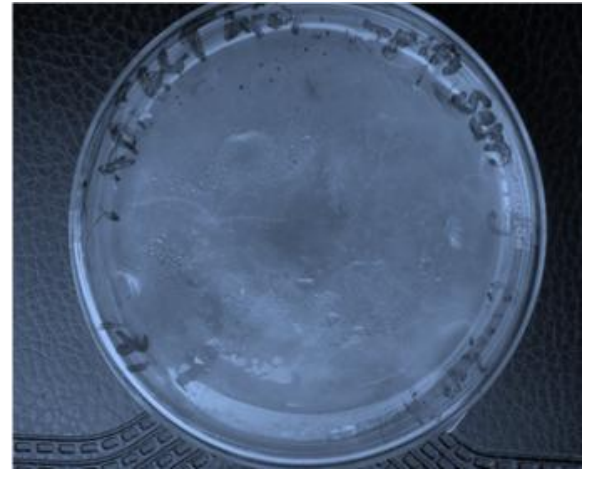

Fig 5: Controlled Bacillus cereus cells showing no growth on oil.

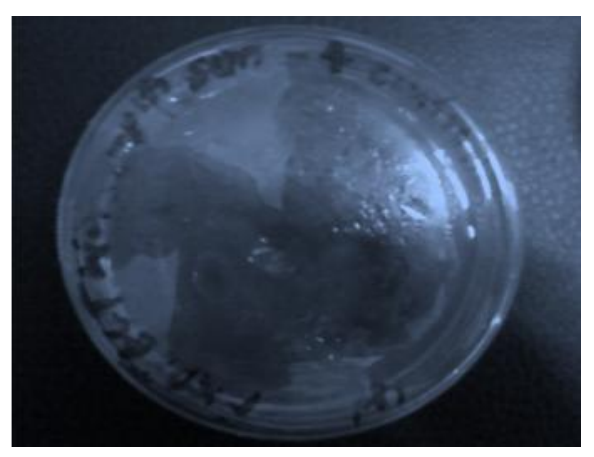

Fig 6: Transformed Bacillus cereus cells showing no growth on oil.

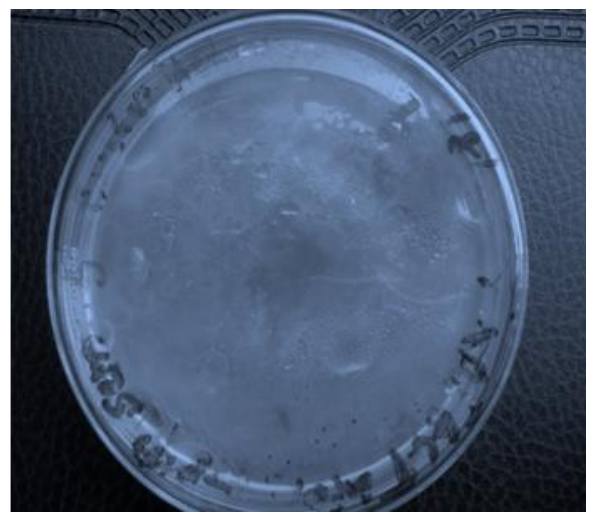

Fig 7: Controlled Bacillus thuringiensis cells showing no growth on oil.

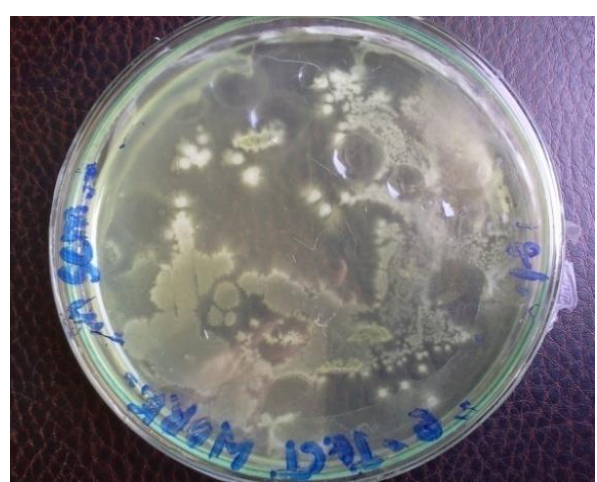

Fig 8: Transformed Bacillus thuringiensis cells showing growth on oil.

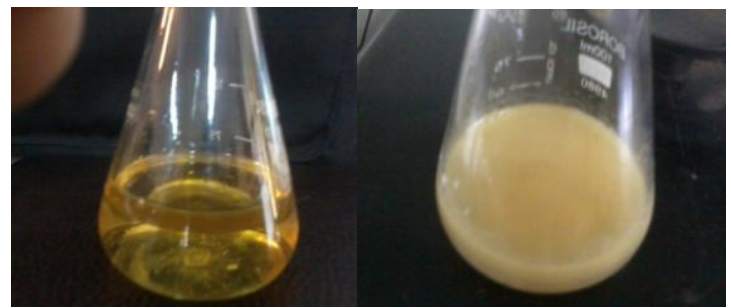

(a)

(b)

Fig 9: (a) Oil layer present on broth just after inoculation of Bacillus thuringiensis, (b) Oil layer disappeared after incubation.

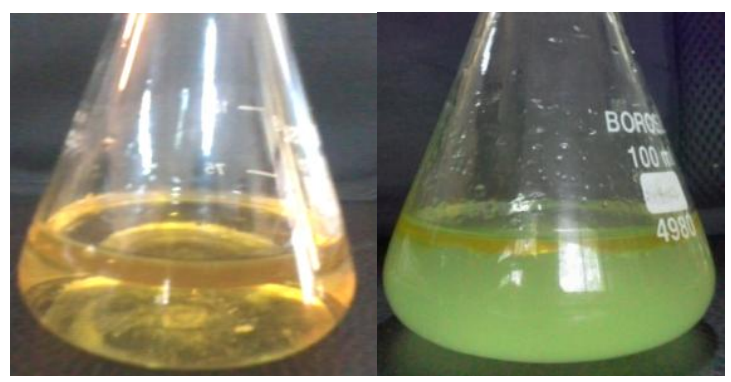

(a)

(b)

Fig 10: (a) Oil layer present on broth just after inoculation of Bacillus cereus, (b) No disappearance of oil layer after incubation.

Table 1: Relationship between Growth of Cells and \% of Degradation

\begin{tabular}{|l|l|l|l|}
\hline $\begin{array}{l}\text { SL } \\
\text { No }\end{array}$ & No. of cells & Time & $\%$ of degradation \\
\hline 1 & 220 & 0 & 0 \\
\hline 2 & 880 & 18 & 11.69 \\
\hline 3 & 968 & 69 & 27.68 \\
\hline 4 & 880 & 126 & 1.43 \\
\hline
\end{tabular}

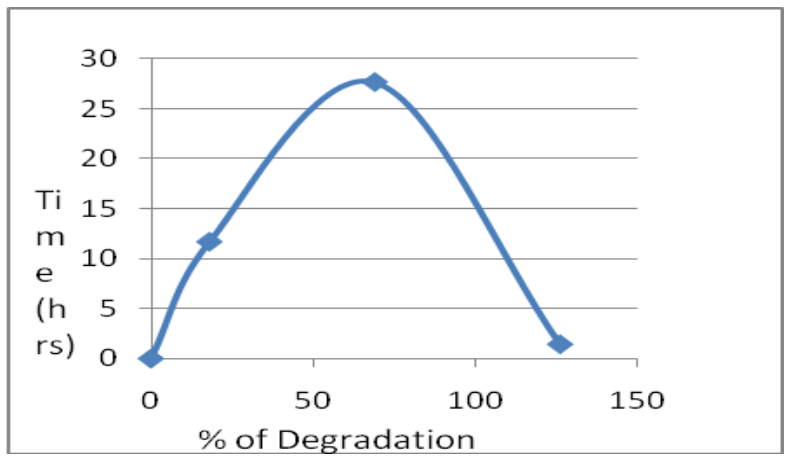

Fig 11: Relationship between time and \% of Degradation 


\section{DISCUSSION}

Pseudomonas aeruginosa is an opportunistic pathogen found ubiquitously in nature. It is one of the most commonly found microorganisms in petroleumcontaminated environments [20]. Studies reveal that Pseudomonas aeruginosa and Pseudomonas putida have the ability to degrade complex compounds [5]. But the pathogenic nature of Pseudomonas aeruginosa makes it unfit for direct use [5]. In the present study the gene responsible for carbon degradation is transferred into Bacillus thuringiensis that can uptake as well as express effectively [8].

Plasmid curing studies have been carried out on Pseudomonas aeruginosa and it was found that they carry catabolic gene for complex carbon compound degradation. Studies conducted by C.Edward Raja et. al revealed the metal resistant property of Pseudomonas where it showed resistance to heavy metals like nickel, cadmium, lead etc [21].

Many methods are in practice for the extraction of DNA among which most of them are time consuming. A homemade kit for plasmid DNA and mini preparation formulated by Simeon Oloni et. al [22] was employed for isolation of plasmid from pseudomonas aeruginosa. Present study employed the same kit and plasmid was isolated successfully.

Studies carried out by Robert A. Spooner et. Al revealed the presence of $x y l R$ and $x l y S$ regulatory sequences in the TOL plasmid Pww0 of Pseudomonas putida which possess the ability to induce catechol-2,3-oxygenase activity. This is a major step in the catabolic pathway [23]. In the present study, comparative studies were carried out between Pseudomonas aeruginosa and Pseudomonas putida where about $40 \%$ similarity was observed by using Pairwise alignment. A match between ORF sequences of both organisms were also seen as a result of which further procedure of molecular cloning was carried out.

Sachiye Inouye conducted molecular cloning of TOC genes $x y l B$ and $x y l E$ of Pseudomonas putida into E.coli for detailed mapping [24]. These are the genes that code for catechol-2,3-oxygenase activity and benzyl alcohol dehydrogenase activity respectively. It was found that the catechol-2,3-oxygenase activity formed in the transformed E.coli carrying $x y l E$ containing plasmid is identical to that formed by the TOL plasmid in Pseudomonas putida. In the present study for transferring the oil degrading capacity of Pseudomonas aeruginosa, transformation of the respective plasmid was carried out into Bacillus thuringiensis.

Qualitative test was carried out by culturing the transformed cells in nutrient broth containing oil. Eventually reduction in the layer of oil was observed and after about 2 weeks complete oil layer was disappeared.

In quantitative studies, drop-weight method was employed to calculate the surface tension where there was a noticeable increase in the surface tension values of broth just after inoculation as well as after a certain period of incubation. Optical density was calculated for both the samples from which the number of cells inoculated and the number of cells responsible for degradation of oil was estimated [19].

\section{CONCLUSION}

Oil spills are one of the major causes of soil and water pollution; hence an efficient and eco friendly method is required to clear it. The study mainly focus on the carbon degrading capacity of Pseudomonas aeruginosa which is encoded by the plasmid DNA but due to pathogenic nature it cannot be directly utilized in the applications hence the plasmid is transformed into Bacillus thuringiensis. A gene called $x y l$ is identified in Pseudomonas putida which encodes for the degradation of complex carbon compounds, this gene sequence along with the sequence of the plasmid of Pseudomonas aeruginosa were compared using pairwise alignment. The plasmid expression studies were carried out qualitatively and quantitatively. Among the two organisms Bacillus thuringiensis and bacillus cereus used Bacillus thuringiensis showed the plasmid expression markably compared to bacillus cereus. Quantative study revealed that the Bacillus thuringiensis degrade $27.68 \%$ of oil with 968 cells in 69 hours. In conclusion, Bacillus thuringiensis with transformed plasmid can be applied towards oil spill treatment. However further standardization and large scale study has to be carried out.

\section{REFERENCES}

[1] John M Pemberton, Radomir Schmidt, Catabolic plasmids-encyclopedia of life sciences (2001, John Wiley \& Sons, Ltd).

[2] Juan-Luis Ramos, Alain Filloux, Pseudomonas volume 5: a model system in biology, Dordrecht Heidelberg London New York, Springer, 2010.

[3] Scott E.Battle, Alan R Houser- Pathogenicity islands of Pseudomonas aeruginosa, US patent US2010/0055702 A1.

[4] Fan Chen, Metabolism of pseudomonas aeruginosa under simultaneous aerobic respiration and denitrification, Doctor of philosophy, The Graduate Faculty of The University of Akron, Dec 2005.

[5] http://www.uptodate.com/contents/epidemiology-andpathogenesis-of-pseudomonas-aeruginosa-infection

[6] Juan-Luis, Alain Filloux-Pseudomonas volume 6: molecular microbiology, infection and biodiversity, Dordrecht Heidelberg London New York, Springer, 2010.

[7] Anne Maczulak, Ph.D- Encyclopedia of microbiology, New York, Facts on file, 2011.

[8] Daniel R. Zeigler, Ph.D, Bacillus genetic stock center catalog of strains, seventh edition, part 2: Bacillus thuringiensis and Bacillus cereus, Bacillus genetic stock center () 1999.

[9] Joanna Kamila Krzeslak, Pseudomonas as a microbial enzyme factory, Department of Pharmaceutical Biology of the University of Groningen, 2009.

[10] Rowland H. Davis, The microbial models of molecular biology: from genes to genomes, Oxford University Press, Inc, 2003.

[11] Piyush Tripathi1, Gopa Banerjee1*, Shivani Saxena1, Mahendra Kumar Gupta1, and P. W. RamtekeAntibiotic resistance pattern of Pseudomonas aeruginosa isolated from patients of lower respiratory tract infection, African Journal of Microbiology Research, 5(19), 23 September, 2011, 2955-2959. 
[12] Frederick M. Ausubel, Roger Brent, Robert E. Kingston, David D. Moore, J.G. Seidman, John A. Smith, Kevin Struhl (eds.), Current Protocols in Molecular Biology, John Wiley \& Sons, 2003.

[13] http://www.ncbi.nlm.nih.gov/

[14] www.ebi.ac.uk/Tools/services/web_emboss_water

[15] http://copicat.dna.bio.keio.ac.jp

[16] Masahiro Yokota, Nobuyoshi Nagamine, Yohei Sugawara, Kris Popendorf, Miho Uchida, Tatsuo Kitahashi, Takashi Komori , Yasubumi Sakakibara Copicat: a software system for predicting interactions between chemical compounds and proteins by using two-layer support vector machine.

[17] Anthony Macalusot \& Anne-Marie Mettus, Efficient Transformation of Bacillus thuringiensis Requires nonmethylated Plasmid DNA, Journal of bacteriology- Feb. 1991, (173), 1353-1356.

[18] M. I. Sinclair and A. F. Morgan, Transformation of Pseudomonas aeruginosa strain Pao with Bacteriophage and plasmid DNA, Aust. J BioI. Sci., 1978, 679-88.

[19] Boon-Beng Lee a , Pogaku Ravindra a \& Eng-Seng Chan, A critical review: surface and interfacial tension measurement by the drop weight method, Chemical Engineering Communications, 195(8), 889-924.
[20] Badrunnisa. S , Manjula Shantaram, Vinitha Ramanath Pai- Isolation, Characterization And Identification Of Bacteria From Coolant Oils, International Journal of Applied Biology and Pharmaceutical Technology, 2(3), July-Sept, 2011, 444-452.

[21] C. Edward Raja, G. S. Selvam, Plasmid profile and curing analysis of Pseudomonas aeruginosa as metal resistant, Int. J. Environ. Sci. Tech., 6 (2), Spring 2009, 259-266.

[22] Simeon Oloni Kotchoni Emma Wanjiru gachomo, Eriola betiku and Olusola Olusoji shonukan - A homemade kit for plasmid DNA mini-preparation, African Journal of Biotechnology, 2(4), April 2003, 88-90.

[23] Robert A. Spooner, Karen Lindsay and F. Christopher H. Franklin- Genetic, functional and sequence analysis of the xylR and xylS regulatory, genes of the tol plasmid pww0, Journal of General Microbiology, 1986, 132, 1347-1 358.

[24] Sachiye Inouye, Atsushi Nakazawa, and Teruko Nakazawa, Molecular cloning of TOL genes xylB and xylE in Escherichia coli, Journal of bacteriology, (145), Mar. 198, 1137-1143. 